\title{
Current Challenges to Achieving a Polio Free World
}

\section{Dear Editor,}

The Expanded Program on Immunisation (EPI), launched in 1974 to protect children against 6 deadly diseases, included the Sabin trivalent oral poliovirus vaccine (tOPV). ${ }^{1}$ This vaccine afforded protection against all serotypes, inducing humoral and mucosal immune response identical to the one induced by wild polioviruses (WPV). tOPV prevents entry of WPV into the central nervous system by the humoral response and blocks WPV replication in mucosal surfaces. ${ }^{2}$ This vaccine also offered important advantages over the Salk inactivated trivalent poliovirus vaccine (IPV), including the augmentation of population immunity by effectively blocking WPV transmission when used in supplemental immunization activities, and its ability to immunise unvaccinated contacts of vaccine recipients by stool shedding of the attenuated virus. ${ }^{2-3}$ Nonetheless, the use of this vaccine had some drawbacks, including its rare ability to cause isolated cases of vaccine-associated paralytic polio (VAPP) and outbreaks of vaccine-derived poliovirus (VDPV). ${ }^{4}$ VAPP is an adverse event following exposure to OPV that occurs approximately 1 in 2.7 million doses of administered OPV. ${ }^{4}$ In contrast, VDPV are polioviruses with atypical genetic properties that pose an important public health concern are the circulating vaccine derived poliovirus (cVDPV), since these viruses circulate in the environment resulting in sustained personto-person transmission, and, owing to their reverted neuro-virulence, produce paralysis similarly to WPV in settings of low OPV coverage. ${ }^{13-14}$ IPV offers protection against paralytic disease by inducing a humoral response but a limited mucosal immunity, reducing its effect in preventing WPV replication in the intestinal mucosa that ultimately hampers its herd protective effect. ${ }^{4}$

By 1988, the World Health Organisation launched the global polio eradication initiative (GPEI) with the overarching goal of eradicating polio by $2000 .^{5}$ Since then, global efforts by the GPEI have reduced wild poliovirus cases by $99 \%$ with more than 2.5 billion polio vaccines administered. ${ }^{3}$ The last human case of wild poliovirus type 2 (WPV2) occurred in 1999, and the last case of wild poliovirus type 3 (WPV3) was identified in 2012 with certification of its eradication on 17 October $2019 .{ }^{6}$ Eradicating polio from the face of the earth requires the elimination the three WPV and c VDPV by achieving and maintaining high levels of population immunity. ${ }^{5}$ By the end of 2019, many countries that had stopped indigenous circulation of WPV were experiencing reintroductions and circulation of cVDPV2. ${ }^{5}$ In 2020, Pakistan had already reported 12 new cases of WPV1, while in 2019, this country and Afghanistan recorded 173 new cases of WPV1, representing a significant increase from previous years. Outside these last strongholds of WPV1 transmission, most polio cases were caused by cVDPV in settings of low OPV immunization coverage in some areas of Africa and Asia, leading to rising waves of concern. ${ }^{5-6}$ Additionally, despite the absence of human cases of WPV in Nigeria, this country has not interrupted the circulation of indigenous WPV 1.5 These events highlight the risk of losing previous gains in polio control efforts, and suggest that the noble goal of achieving a world free of polio remains elusive despite billions of dollars spent. ${ }^{3,5}$

More recently, after the certification of the eradication of WPV2 poliovirus in 2015, the GPEI decided to remove the poliovirus type 2 antigenic component from the oral polio vaccine as an essential step towards a poliofree world. ${ }^{7-8}$ This important step was taken because of the potential risk of OPV2 in causing paralytic polio in immunocompromised children, and to improve the immunogenicity of this monovalent vaccine..$^{7-8} \mathrm{~A}$ globally coordinated switch to bivalent OPV (bOPV) containing only attenuated viruses type 1 and type 3 requires the previous introduction of IPV in highrisk countries. ${ }^{7-8}$ This vaccination strategy attempts to reduce the risks of outbreaks of cVDPV2 associated with the withdrawal of OPV2, and to boost immunity to the other serotypes. ${ }^{3}$ However, this globally synchronized switch, in April 2016, occurred at a time when IPV coverage was suboptimal in many settings. ${ }^{6}$ As a result of this switch, the number of children with reduced immunity to WPV2 grew exponentially, allowing the introduction of cVDPV2 in many countries since the switch took place. ${ }^{5-6}$ Unfortunately, in many of these countries, the outbreaks of cVDPV2 that subsequently 
occurred have coincided with an overall low OPV immunisation coverage. The need for reactive mass vaccination efforts with the use of monovalent OPV2 vaccine illustrates the ecological constraints and selective pressure that fuels the transmission of this particular polio serotype.

The recent upsurge of both of WPV1 in Afghanistan and Pakistan in 2019 and the increasing number of cases in Pakistan in 2020, as well as the rising number of outbreaks of cVDPV2 call for a renewed approach. ${ }^{9-15}$ Polio vaccination efforts should be coordinated by existing EPI surveillance and operational platforms with sufficient resources allocated for effective vaccine delivery. ${ }^{3}$ Vaccination efforts should be part of daily routine activities of health facilities at all levels in a country, using immunisation strategies that include microplanning of activities with a consistent, regular, and rigorous review of surveillance data. ${ }^{15}$ Eventually, withdrawing the use of all forms of OPV and introducing the use of IPV will ensure long-term immunity and prevent future cVDPV outbreaks. ${ }^{6}$ There is also a growing consensus that the use of IPV in all settings during routine and supplementary immunisation activities offers the most likely path to achieve the global eradication of polio, given its impact in reducing paralytic polio and by its ability to foster mucosal immunity among those with a previous history of OPV vaccination. ${ }^{7-8}$

The inability to bring global cases to zero highlights the realities of modern times, including geopolitical barriers and conflicts, the increasing connectivity provided by social media and the $24 / 7$ news cycle outlets. ${ }^{10-13}$ These confluent forces are increasing the public's distrust and misinformation about vaccination ${ }^{4}$ as exemplified by the controversies with dengue vaccine introduction, and HPV vaccine implementation in different settings. ${ }^{9-12}$ Adopting communication strategies that promote clarity of purpose and relevance to community needs may potentially improve immunisation uptake and reduce vaccine rejection and vaccine hesitancy. ${ }^{10-12}$

There is also an important need to incorporate local knowledge and expertise into designing delivery strategies, so as to improve operational immunisation activities targeting hard-to-reach communities. ${ }^{12-15}$ The resurgence of polio cases in Pakistan is a result of multiple factors; a significant one is community and healthcare worker fatigue, leading to decreased accountability and ownership. ${ }^{12,14}$ Counter-insurgency operations carried out by the US involvement in Pakistan with the use of drone strikes, and the use of fake vaccination programmes to gather intelligence for the assassination of Osama bin Laden in 2011, have intensified disruption efforts of polio immunisation campaigns by militants. ${ }^{9}, 14$ These interventions have also contributed to the public's mistrust of polio vaccination efforts in Pakistan, Afghanistan and other countries in this region. ${ }^{9-12}$ Despite substantial financial investments, polio programme activities have not substantially enhanced staff capacity or sustained morale among programme staff. ${ }^{14-15}$ The verticality of the polio programme has exacerbated programmatic deficits in Afghanistan that, along with the ongoing civilian unrest, have left clusters of children unvaccinated in many provinces. Among these last strongholds of polio transmission, there is a need to pay close attention to the missing voice of the affected communities in order to overcome community fears and to prevent fatigue and complacency. ${ }^{9}$

Poliovirus infection is one of the many public health priorities facing many human groups living in low-resource settings. Therefore, understanding the perceptions and beliefs of affected communities and actively engaging those affected, offer the best opportunity for long-term sustainable benefits. We strongly suggest that empowering and reducing social inequities among disenfranchised populations living in the last strongholds of polio transmission would be a pivotal factor in potentiating current eradication efforts.

In addition to the above concerns, the current pandemic of SARS-CoV-2 brings a new set of concerns regarding routine immunisation activities and polio control efforts. In late December 2019, in Wuhan China, the Severe Acute Respiratory Syndrome Coronavirus 2 (SARS-CoV-2) emerged as a new viral pathogen causing the Coronavirus Disease 2019 (COVID-19), which causes significant morbidity and mortality, especially in persons above 55 years of age and among those with chronic medical conditions. Globally, human infections due to SARS-CoV-2 have occurred in more than 187 countries, with more than 5 million cases and 330,000 deaths. Without a doubt, national, regional, and international efforts to contain the COVID-19 may delay timelines in achieving immunisation goals, given the mandatory social distancing interventions instituted in most countries to contain the outbreak, along with economic and social disruptions. We should expect that polio control efforts will be impacted by the diversion of resources, allocation of public health personnel, and political commitments. Only time will tell how much polio control activities and other crucial immunisation practices are affected by the global disruption caused by the ongoing SARS-CoV-2 pandemic. 


\section{REFERENCES}

1. Chan M. Beyond expectations: 40 years of EPI. Lancet 2014 May 17; 383(9930): 1697-8.

2. Parker EPK, Molodecky NA, Pons-Salort M, O’Reilly KM, Grassly NCC. Impact of inactivated poliovirus vaccine on mucosal immunity: implications for the polio eradication endgame. Expert Rev Vaccines 2015; 14(8): 1113-1123.

3. Cochi SL, Hegg L, Kaur A, Pandak C, Jafari H. The Global Polio Eradication Initiative: Progress, lessons learned, and polio legacy transition planning. Health Aff (Millwood) 2016 Feb; 35(2): 277-83.

4. Burns CC, Diop OM, Sutter RW, Kew OM. Vaccine-derived polioviruses. J Infect Dis 2014 Nov; 210 (Suppl 1): S283-S293.

5. Polio Eradication Initiative. Available at: http://polioeradication.org/ polio-today/polio-now/this-week/ Accessed on 21 May 2020.

6. Pons-Salort M, Burns CC, Lyons H, Blake IM, Jafari H, Obserte MS, Kew OM, Grassly NC. Preventing vaccine-derived poliovirus emergence during the polio endgame. PLoS Pathog 2016 Jul 6; 12(7): e1005728.

7. John TJ, Dharmapalan D. An ethical appraisal of the choice of vaccine against poliomyelitis. Indian J Med Ethics Jan-Mar 2019; 4(1) NS:26-9.

8. Agarwal RK. Polio eradication in India: A tale of science, ethics, dogmas and strategy. Indian Pediatr 2008 May;45(5):349-51.

9. Peckham R. Polio, terror and the immunological worldview. Glob Public Health 2018 Feb;3(2):189-210.

10. Wang Y, McKee M, Torbica A, Stuckler D. Systematic literature review on the spread of health-related misinformation on social media. Soc Sci Med 2019 Nov;240:112552.

11. Dubé E, Vivion M, MacDonald NE. Vaccine hesitancy, vaccine refusal and the anti-vaccine movement: influence, impact and implications. Expert Rev Vaccines 2015 Jan;14(1):99-117.
12. Ali M, Ahmad N, Khan H, Ali S, Akbar F, Hussain Z. Polio vaccination controversy in Pakistan. Lancet 2019 Sep 14;394:915-6.

13. Franco-Paredes C. Reconstructing the past of poliovirus eradication efforts. Lancet Infect Dis 2010 Oct; 10(10):660-1.

14. Nadeem NJ. The global polio eradication initiative (GPEI) in Pakistan. J Pak Med Assoc. 2016 Nov;66(11):1466-71.

15. Ong BKC, Fisher DA. Infectious Diseases Eradication: Poliomyelitis as a lesson in why "close" is not good enough. Ann Acad Med Singap 2010; 39(7): 532-10.

Carlos Franco-Paredes, ${ }^{1,2} \mathrm{MD}, \mathrm{MPH}$,

Andres Henao-Martinez, ${ }^{1}{ }_{M D}$, Lakshmi Chauhan, ${ }^{1} M D, M S c$, Alfonso J Rodriguez-Morales, ${ }^{3,4}{ }_{M D}$, M Imran Khan, ${ }^{5} M D$

${ }^{1}$ Division of Infectious Diseases, Department of Medicine, University of Colorado Anschutz Medical Center, Aurora CO, USA.

${ }^{2}$ Hospital Infantil de México Federico Gómez, México City, México.

${ }^{3}$ Committee on Tropical Medicine, Zoonoses and Travel Medicine, Colombian Association of Infectious Diseases (ACIN), Bogota, Colombia

${ }^{4}$ Public Health and Infection Research Group, Faculty of Health Sciences, Universidad Tecnológica de Pereira, Pereira, Risaralda, Colombia. PHC Global (Pvt.) Ltd, Bahadurabad 3, Karachi, Pakistan

Address for Correspondence: DrCarlos Franco-Paredes, Division of Infectious Diseases, University of Colorado, Anschutz Medical Center Email: carlos.franco-paredes@cuanschutz.edu 